\title{
Monitoreo de instalación fotovoltaica usando bus CAN
}

\section{Photovoltaic installation monitoring using CAN bus}

\author{
LUJÁN-RAMÍREZ, Carlos Albertợ, SANDOVAL-GÍO, Jesús*, MORENO-SABIDO, Mario Renán, y \\ ATOCHE-ENSEÑAT, José Ramón
}

\section{Tecnológico Nacional de México / I.T. Mérida}

ID $1^{\text {er }}$ Autor: Carlos Alberto, Luján-Ramírez / ORC ID: 0000-0002-8978-9188, Researcher ID Thomson: T-6838-2018, arXiv Author ID: clujan, CVU CONACYT ID: 296592

ID $1^{\text {er }}$ Coautor: Jesús, Sandoval-Gío / ORC ID: 0000-0001-5847-3669, Researcher ID Thomson: V-1930-2018, arXiv Author ID: jesus.sandoval, CVU CONACYT ID: 297308

ID $2^{\text {do }}$ Coautor: Mario Renán, Moreno-Sabido / ORC ID: 0000-0003-4690-7583, Researcher ID Thomson: T-3397-2018, arXiv Author ID: xacdc12, CVU CONACYT ID: 406193

ID $3^{\text {er }}$ Coautor: José Ramón, Atoche-Enseñat / ORC ID: 0000-0002-0214-7335, Researcher ID Thomson: Q-2005-2018, arXiv Author ID: jratoche, CVU CONACYT ID: 200715

DOI: $10.35429 /$ JRE.2020.12.4.32.40

Recibido 03 de Abril, 2020; Aceptado 30 de Junio, 2020

\section{Resumen}

Este artículo aborda el problema de desarrollar un sistema para monitorear el comportamiento de módulos solares usando una red CAN. Se desea medir corriente, voltaje, y temperatura en distintas condiciones de operación de una instalación fotovoltaica, con el fin de obtener la información necesaria para posteriormente usarla en la determinación de su eficiencia energética. En el sistema propuesto, los datos de corriente, voltaje y temperatura son transmitidos a través una red CAN basada en dispositivos de la familia de microcontroladores de Microchip®. El diseño de la red se conforma de nodos esclavos encargados de llevar a cabo la adquisición de datos y conducirlos a un nodo maestro CAN Central. Este nodo maestro los recibe, se encarga de la temporización de las tareas y se conecta vía puerto serial a una computadora personal. El entorno de programación utilizado es el de MikroC® para dsPIC®. El proceso de envío de los datos se puede observar a través de la terminal USART del MikroC®, éstos se guardan en un archivo .txt para su posterior análisis con algún programa científico. Se reportan pruebas experimentales realizadas con un grupo de paneles comerciales bajo dos condiciones de operación: corto circuito y circuito abierto. Se muestran las gráficas, y para validar la información que aportan los datos adquiridos se verifican las relaciones entre las variables monitoreadas considerando el conocimiento obtenido de la literatura.

Monitoreo paneles solares, dsPIC, Red CAN

\begin{abstract}
This paper addresses the problem of developing a system to monitor the behavior of solar modules using a CAN network. It is desired to measure current, voltage, and temperature under different operating conditions of a photovoltaic installation, in order to obtain the necessary information to later use it to determine its energy efficiency. In this work, current, voltage and temperature data are transmitted over a CAN network based on devices from the Microchip ${ }^{\circledR}$ family of microcontrollers. The network design is made up of slave nodes in charge of carrying out data acquisition and leading them to a CAN Central master node. This master node receives them, oversees timing tasks and connects via serial port to a personal computer. The programming environment used is MikroC $®$ for dsPIC $®$. The process of sending the data can be observed through the MikroC® USART terminal, these are saved in a .txt file for later analysis with a scientific software. Experimental tests carried out with a group of commercial panels are reported under two operating conditions: short circuit and open circuit. The graphs are shown, and to validate the information provided by the acquired data, the relationships between the monitored variables are verified considering the knowledge obtained from the literature.
\end{abstract}

Solar panels monitoring, dsPIC, Can Bus

Citación: LUJÁN-RAMÍREZ, Carlos Alberto, SANDOVAL-GÍO, Jesús, MORENO-SABIDO, Mario Renán, y ATOCHEENSEÑAT, José Ramón. Monitoreo de instalación fotovoltaica usando bus CAN. Revista de Energías Renovables. 2020. 412: $32-40$

\footnotetext{
* Correspondencia del Autor (Correo electrónico: jesus.sg@merida.tecnm.mx)

$\dagger$ Investigador contribuyendo como primer autor.
} 


\section{Introducción}

Una instalación fotovoltaica está formada por varios módulos solares que se conectan entre sí, por ejemplo, usando un grupo de microinversores en una instalación conectada a la red eléctrica o a un controlador de carga de baterías en un sistema aislado. La eficiencia energética de conversión de la instalación fotovoltaica depende de su punto de operación, el cual está determinado por ciertas variables como la cantidad de irradiación solar que recibe, la orientación del módulo respecto a esta radiación, así como de la temperatura en la que opera y las condiciones particulares de su sistema de carga (So, Jung, Yu, \& Choi, 2007).

Es tema de gran interés determinar cuál es la eficiencia energética con el que se desempeña una instalación solar particular bajo diversas condiciones de operación (Li Vigni, y otros, 2015). Una contribución al abordaje de este problema es diseñar un sistema que pueda proporcionar los datos más importantes que se relacionan con el rendimiento de esa instalación. Estos son, en términos generales, la temperatura $\mathrm{T}$ y la irradiancia $\mathrm{G}$, es decir, la cantidad de irradiación solar que recibe. Estos dos parámetros se presentan usualmente en las curvas características I-V y P-V, incluidas en las hojas de datos del fabricante de los módulos solares. Sin embargo, estas curvas solo son válidas bajo condiciones estándar de operación (SC) y cambian de forma conforme las condiciones ambientales progresan a lo largo del día (Castillo Atoche, y otros, 2015). Es deseable disponer de un sistema que pueda recabar suficiente información en tiempo real que pueda usarse para realizar un análisis del performance de la instalación (So, Jung, Yu, \& Choi, 2007).

Dentro del ámbito del monitoreo de una instalación fotovoltaica se emplean distintos métodos, los cuales incluyen soluciones alámbricas e inalámbricas (Papageorgas, y otros, 2013), (Xie, Yang, Zhang, \& Li, 2015), (Kang, Kang, \& Choi, 2013), y (Rivai \& Rahim, 2013). Los sistemas basados en redes inalámbricas orientan su aplicación a sistemas de grandes dimensiones y alejados de los centros de control, destinados, la mayoría de las veces, a satisfacer al usuario no experto en el tema que dispone de un dispositivo móvil y una conexión a internet. Son sistemas relativamente sensibles al ruido eléctrico y de limitada velocidad (Sarswat, Yadav, \& Maurya, 2019) (Kekre \& Gawre, 2017).
Por otro lado, los sistemas alámbricos se prefieren cuando los arreglos no son muy extendidos y se requiere mayor velocidad e inmunidad al ruido (Mohanraj, Thottungal, \& Jaikumar, 2013).

Uno de los buses de campo más usados en la industria es el CAN (Controller Area Network) CAN tiene diferentes capas físicas que puede utilizar. La capa física más común es la CAN de alta velocidad. Esta está implementada con dos cables y permiten la comunicación con tasas de transferencia de hasta 1 Mbps. En una red CAN es posible interconectar hasta 110 nodos sin afectar la velocidad de transmisión (Bosch, 1991). Así, en la aplicación con una instalación fotovoltaica, es posible usar un nodo como elemento interfaz en la adquisición de los datos de cada módulo solar. Como se mencionó, CAN simplifica mucho el cableado ya que utiliza solamente 2 líneas de transmisión denominadas CAN Low y Can Hight.

En este trabajo se describe un sistema de adquisición de datos fiable, diseñado e implementado para medir y monitorear en tiempo real, las características más importantes que se relacionan con el rendimiento de un módulo solar, y/o sistema, haciendo uso de las ventajas de las redes CAN. Según (Núñez, Benítez, Proenza, Vázquez, \& Diaz, 2020) las pérdidas de energía que ocurren en un sistema fotovoltaico pueden deberse principalmente a dos causas: las pérdidas operacionales y los fallos. Las primeras, que están siempre presentes en cualquier sistema fotovoltaico, se relacionan con las diferencias que existen en el ámbito práctico, entre la eficiencia nominal y la real.

Aquí entran las pérdidas de energía resistiva en los cables de conducción eléctrica y las pérdidas debido a la degradación de los componentes por efecto del ambiente y el envejecimiento. Los fallos están asociados al mal funcionamiento de, al menos, un componente del sistema y, en consecuencia, por salir de sus condiciones típicas de operación.

El sistema propuesto se utiliza para adquirir información de las condiciones de operación del sistema fotovoltaico, midiendo datos en el módulo solar conectado a la red y transferir los valores registrados a una computadora de supervisión para su almacenamiento y posterior procesamiento. 
El alcance de este sistema de monitoreo va más allá de mostrar solamente una gráfica en una interfaz de usuario.

La presentación de este trabajo se organiza de la siguiente manera:

1. Materiales. Se muestran los materiales y recursos tecnológicos empleados en el diseño del sistema de monitoreo.

2. Método. Se precisa el método empleado en el diseño e implementación del prototipo.

3. Resultados. Se detalla el procedimiento experimental para comprobar el adecuado funcionamiento del sistema.

4. Conclusiones. Se destacan las conclusiones alcanzadas en esta investigación.

5. Agradecimientos. Se mencionan las colaboraciones que apoyaron el desarrollo del proyecto.

\section{Materiales}

\section{EI bus CAN}

El bus CAN es uno de los buses de campo más utilizado actualmente. El protocolo CAN fue diseñado por Bosch en 1986 para aplicaciones de la industria automotriz como una forma de lograr una comunicación serial de gran robustez. (Bosch, 1991). A partir de 1994, se han desarrollado y estandarizado otros protocolos de alto nivel a partir de CAN, como CANopen ${ }^{\circledR}$ y DeviceNet ${ }^{\circledR}$, y su uso se ha extendido a otras industrias. Mediante la implementación de una red CAN se pueden comunicar varias estaciones con otras sin el inconveniente de agregar carga computacional al controlador principal.

Cada estación de la red puede funcionar como dispositivos de sensado, actuación, despliegue de información, controladores, entre otras cosas. (Mohanraj, Thottungal, \& Jaikumar, 2013). Las principales características de la comunicación mediante bus CAN incluyen una velocidad de hasta $1 \mathrm{Mbps}$, esquema multimaestro, con capacidad de autodiagnóstico y reparación de errores. La arquitectura distribuida de la red permite fácil mantenimiento y bajo costo.
Una de las más apreciadas ventajas es la alta inmunidad al ruido de interferencia electromagnética ya que emplea un alambrado con señales diferenciales con dos niveles de voltaje: un '0' lógico dominante y un ' 1 ' lógico recesivo. No existe una línea específica de reloj para generar sincronización; ésta se obtiene a partir de los flancos de las propias señales.

\section{Controlador digital de señales dsPIC}

El Circuito Integrado (CI) dsPIC30f4013, es un Controlador de Señales Digitales (DSC) a 16 bits de la familia Microchip ${ }^{\circledR}$ con una arquitectura de alto rendimiento basada en RISC ( Microchip Technology Incorporated, 2007).

Los dsPIC se comenzaron a producir a gran escala a finales de 2004. Son los primeros PIC con buses de datos de 16 bits. Reúnen todas las posibilidades de los anteriores PIC y añaden varias operaciones de DSP (Digital Signal Processing) implementadas en hardware, como multiplicación con suma de acumulador (multiply-accumulate, MAC), barrel shifting, bit reversion o multiplicación 16x16 bits.

Algunas características del dsPIC30f4013 se presentan en la Tabla 1.

\begin{tabular}{|l|l|}
\hline \multicolumn{1}{|c|}{ Característica } & \multicolumn{1}{c|}{ Cantidad } \\
\hline Eeprom & $1024 \mathrm{~B}$ \\
\hline Sram & 2KB \\
\hline Memoria De Programa & $48 \mathrm{~KB}$ \\
\hline Entradas/Salidas & 30 \\
\hline Timers & 5 de $16 \mathrm{bits}$ \\
\hline Adc & 12 bits, 200 ksps y 13 canales \\
\hline & CAN \\
Interfaz & I2C \\
& SPI \\
& UART x2 \\
\hline
\end{tabular}

Tabla 1 Características del DSPIC30F4013. Fuente: dsPIC30F3014/4013 Data Sheet. Microchip Technology Inc. 2010

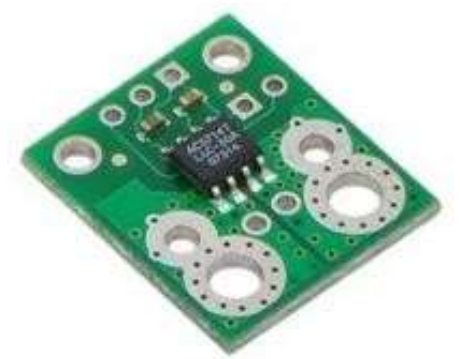

Figura 1 Sensor de corriente de efecto Hall, modelo ACS714

Fuente: www.pololu.com 


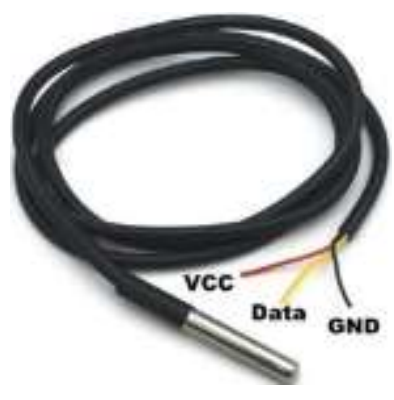

Figura 2 Sensor de temperatura, modelo DS18B20 Fuente: Maxim Integrated Productos Inc.

\section{Monitor de tensión}

El circuito integrado que se utilizó para la monitorización del voltaje en cada módulo fotovoltaico es el INA114AP, que es un amplificador de instrumentación que ofrece una excelente precisión (Burr-Brown Corporation, 2020). El proceso de muestreo está basado en un simple divisor de tensión, aprovechando la alta impedancia del amplificador de instrumentación.

\section{Monitor de corriente}

Para obtener el valor de la corriente que produce cada módulo solar, se utilizó el sensor de corriente ACS714 de -30 amperes a +30 amperes. En la Figura 1 se muestra el modelo del sensor de corriente ACS714. Este dispositivo utiliza el principio del efecto Hall para sensar la intensidad de la corriente que genera cada módulo fotovoltaico, proporcionando un valor de voltaje proporcional a la corriente que circula a través de este. De acuerdo con (Allegro, 2013), la sensibilidad en la salida es de 66 a 185 mv/A.

\section{Monitor de temperatura}

Se utiliza el sensor de temperatura de precisión DS18B20. Este proporciona lecturas con una resolución de 9 a 12 bits (Maxim Integrated Products, Inc., 2019). Como puede verse en la Figura 2, presenta 3 terminales (VCC, GND, y Data). El encapsulado del sensor tiene un cuerpo metálico que es ideal para utilizarlo en exteriores. La comunicación se hace a través de una sola línea (One Wire Bus), es decir, se requiere de una sola línea de datos. La colocación del sensor se ubica en la parte posterior de la estructura de los paneles.

\section{Metodología \\ Diseño del sistema}

El sistema se construye a partir del diagrama a bloques que se presenta en la Figura 3. Cada elemento de la red se construye a alrededor del Circuito Integrado (CI) dsPIC30f4013.

Para utilizar el bus CAN en el dsPIC, se debe conectar un transceptor ya que este DSC no lo integra. Dentro de la gama básica de integrados de Microchip, está el MCP2551 (Microchip Technology Inc., 2007). Este es un transceptor CAN de alta velocidad que puede funcionar a velocidades de hasta 1 Mbps.

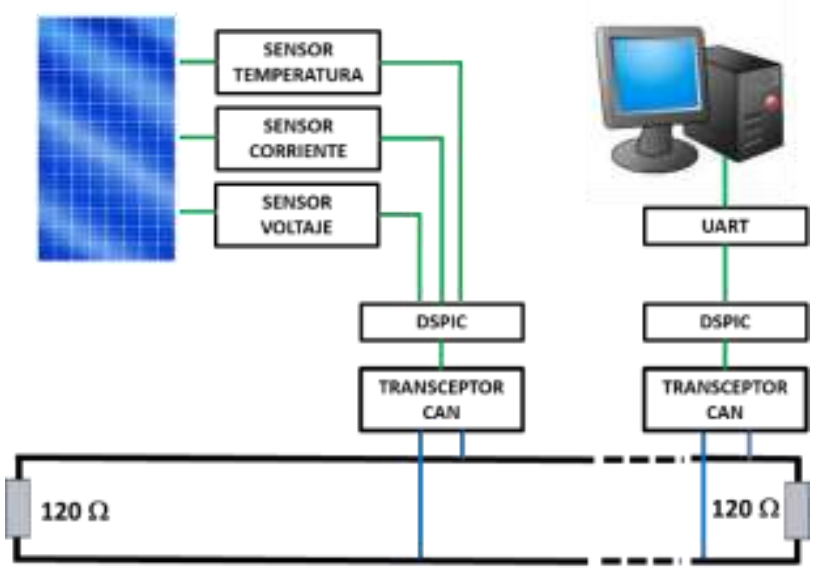

Figura 3 Esquema general del sistema Fuente: Elaboración Propia

El MCP2551 sirve de interfaz entre un controlador CAN y el bus físico y proporciona capacidad de transmisión y recepción diferencial, además de ser completamente compatible con ISO 11898. Adicionalmente es adecuado para sistemas de $12 \mathrm{~V}$ y $24 \mathrm{~V}$. Posee entrada externa de control en pendiente para reducir las emisiones RFI, implementa los requisitos de capa física ISO 11898, detección dominante permanente, un funcionamiento en espera de baja corriente y una alta inmunidad al ruido gracias a la implementación de un bus diferencial.

Es necesario un transceptor para cada nodo (ver Figura 4). El funcionamiento es el siguiente: el módulo CAN se implementa en el microcontrolador como una interfaz serie, utilizada para la comunicación con otros módulos CAN u otros dispositivos del propio microcontrolador (Stoneking, 2002). 
Para interconectar a la vez todos los nodos de la red en un mismo punto, es necesario crear terminales utilizando jacks RJ-45. Solo se conectan 2 pines comunes en todas y cada una de las terminales, de correspondencia a las líneas CAN Low y CAN High.

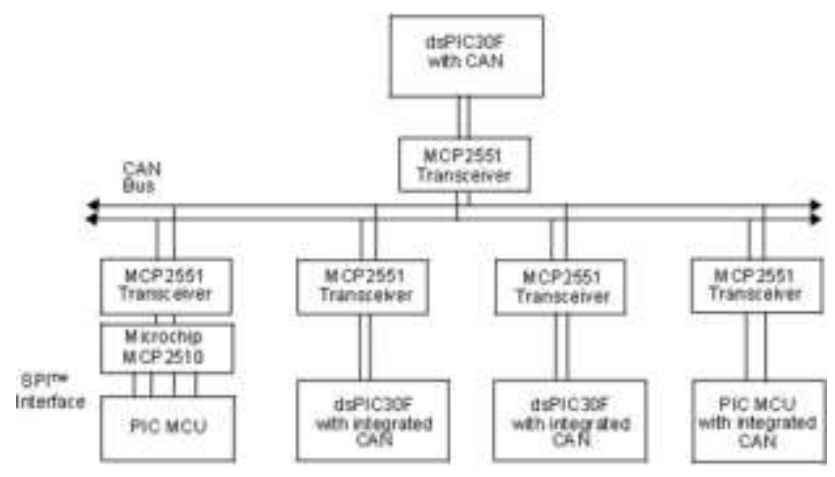

Figura 4 Implementación de la red CAN usando dispositivos de la familia PIC

Fuente: Microchip Inc

La programación de los dispositivos se realizó con mikroC PRO®. Este es un completo compilador de ANSI C para los dispositivos PIC de Microchip ${ }^{\circledR}$ desarrollado por MikroElektronika®. Es una excelente solución para el desarrollo de códigos, es un ambiente de desarrollo con editor de texto, bibliotecas con múltiples funciones para diversos módulos y herramientas incorporadas para agilizar enormemente el proceso de programación.

\section{Pruebas experimentales}

Las pruebas experimentales se llevaron a cabo en el Laboratorio de Energías Renovables de la Facultad de Ingeniería de la Universidad de Yucatán con coordenadas: Latitud: $21^{\circ} 02^{\prime} 54^{\prime \prime}$ Norte, Longitud: 89 38' 35" Oeste, Altura: 6,27 msnm. Durante el desarrollo experimental se emplearon 4 paneles fotovoltaicos del tipo Sunmodule SW 50 poly RMA.

La Tabla 2 muestra los datos de la hoja del fabricante (Solar World AG, 2012), bajo condiciones estándar de prueba (STC: $\mathrm{G}=1000 \mathrm{~W} / \mathrm{m}^{2}, \mathrm{~T}=25^{\circ} \mathrm{C}, \mathrm{AM} 1.5$ ).

Se espera una reducción de la eficiencia en el comportamiento con carga parcial a $25^{\circ} \mathrm{C}$ : A 200 $\mathrm{W} / \mathrm{m}^{2}$ se alcanza el $95 \%(+/-2 \%)$ de la eficiencia bajo STC.

\begin{tabular}{|l|l|l|l|}
\hline \multicolumn{1}{|c|}{ Característica } & \multicolumn{1}{c|}{ Símbolo } & Valor & Unidades \\
\hline Máxima potencia & Pmax & 50 & $\mathrm{~W}$ \\
\hline $\begin{array}{l}\text { Voltaje a circuito } \\
\text { abierto }\end{array}$ & Voc & 22.1 & $\mathrm{~V}$ \\
\hline $\begin{array}{l}\text { Voltaje en el punto } \\
\text { de máxima potencia }\end{array}$ & $\mathrm{Vmpp}$ & 18.2 & $\mathrm{~V}$ \\
\hline $\begin{array}{l}\text { Corriente de corto } \\
\text { circuito }\end{array}$ & Isc & 2.95 & $\mathrm{~A}$ \\
\hline $\begin{array}{l}\text { Corriente en el punto } \\
\text { máximo de potencia }\end{array}$ & Impp & 2.75 & $\mathrm{~A}$ \\
\hline
\end{tabular}

Tabla 2 Características generales del panel solar Sunmodule SW 50 poly RMA Data sheet

Fuente: SolarWorld AG. 5/2010

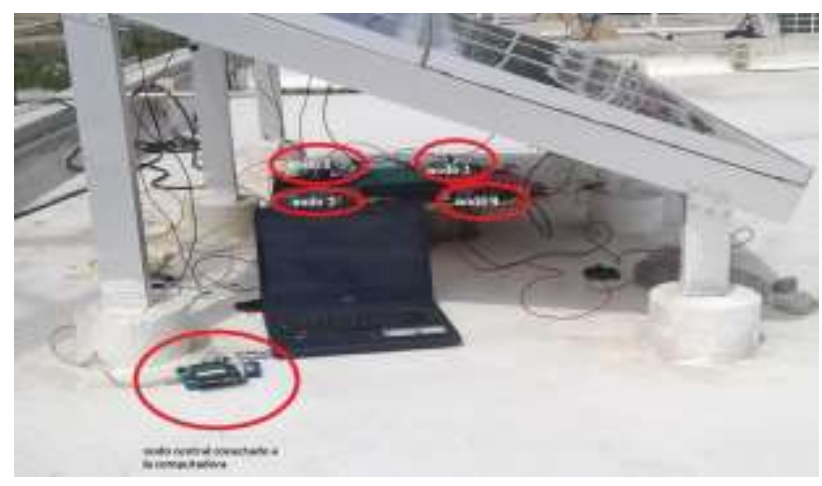

Figura 5 Implementación de la red CAN en cuatro módulos de 50W en la Facultad de Ingeniería de la Universidad Autónoma de Yucatán

Fuente: Elaboración Propia

En la Figura 5 se muestra la instalación de todo el sistema que constituye la Red CAN.

Cabe recordar que el sensor de temperatura se adapta a la parte posterior de cada panel, la salida de voltaje de cada panel en la entrada del circuito divisor de voltaje en la tarjeta de adquisición, el sensor de corriente en serie con alguna carga, que puede ser una resistencia variable de potencia, y el nodo central a la computadora.

El día 14 de abril de 2019 se hizo la primera medición comenzando a las 6:00 horas y concluyendo a las 20:00. Se tomaron lecturas cada 5 minutos y se observaron dos condiciones de operación: salida de los módulos en corto circuito y circuito abierto. Estas condiciones se operaron desde los dsPIC usando un circuito de control formado por un relevador y un transistor MOSFET de potencia conectados a un puerto digital. El nodo central va solicitando a cada nodo esclavo los datos de temperatura, tensión y corriente en intervalos de tiempo de cinco minutos. Se permite un tiempo de entre uno y dos minutos para la estabilización térmica del panel acoplado a la respectiva carga en cada proceso de medida. 
El nodo central dispone de un LCD donde se puede apreciar la medición de temperatura en valor decimal, el de voltaje y la corriente. Adicionalmente, se tomaron medidas de la irradiancia en forma paralela con un piranómetro desarrollado previamente y descrito en (Sandoval Gío, Luján Ramírez, Sandoval Curmina, \& Chan Sosa, 2018).

Al hacer la medición el día 18 de abril del 2019, la temperatura promedio de los 4 módulos solares fue de $40.65^{\circ} \mathrm{C}$, mientras que el voltaje promedio fue de $19.05 \mathrm{v}$, y de acuerdo con la Tabla 2 que muestra el voltaje a circuito abierto en condiciones estándar, es decir, a $25^{\circ} \mathrm{C}$ ofrece 22.1 volts. Entonces, se puede concluir, que el incremento de la temperatura en un módulo solar reduce la tensión de salida en circuito abierto Voc.

Una característica importante es que se pueden conectar/desconectar nodos a la red sin afectar el funcionamiento de los demás. Hay que considerar, sin embargo, que conforme aumenta la distancia, disminuye la velocidad del bus. La velocidad máxima del bus CAN se obtiene a 40 metros utilizando en los extremos terminales dos resistencias de $120 \Omega$, de acuerdo con el ISO 11898-2.

\section{Resultados}

A partir de los experimentos realizados se procedió validar los datos obtenidos en la medición de un panel fotovoltaico relacionando las diferentes variables en dos condiciones de operación: el panel en corto circuito y en circuito abierto. Existe amplia información en la literatura que desarrollan extensamente las relaciones anteriormente citadas (Xiao, $\mathrm{Yu}$, Yang, \& Que, 2014), (Löper, y otros, 2012). Se muestran a continuación las gráficas obtenidas en este procedimiento experimental.

Se observa en la Gráfica 1 la evolución de la temperatura $\mathrm{T}\left(\mathrm{en}{ }^{\mathrm{O}} \mathrm{C}\right)$ y la irradiancia $\mathrm{G}(\mathrm{en}$ $\mathrm{W} / \mathrm{m}^{2}$ ) en diferentes momentos del día.

En la Gráfica 2 se muestra la muy cercana relación entre la irradiancia $\mathrm{G}$ y la corriente de corto circuito Isc, ya descrita por otros autores en la literatura (Kaundal, Mondal, Sharma, \& Bansal, 2015). La curva de dispersión de la Gráfica 3 demuestra con claridad esta dependencia.
La poca variación de la tensión Voc con la irradiancia G se ilustra en la Gráfica 4.

La relación entre Voc y G se describe en (Shariar, Mohammed, Rahman, \& Islam, 2011), y puede observarse en la Gráfica 5.

La Gráfica 6 muestra el efecto de relación entre Voc y la temperatura T durante el intervalo de tiempo en el que la irradiancia tiene una poca variación relativa, esto es, entre las 12:00 y las 14:30 hrs. Este efecto de dependencia inversa se encuentra documentado en (Shariar, Mohammed, Rahman, \& Islam, 2011) y (Löper, y otros, 2012).

Dada la programabilidad del sistema, es posible rediseñar los experimentos para diferentes escenarios que destaquen por su mayor complejidad, los cuales son tópicos de gran interés para la investigación de varios autores. Ver a (Prieto, Pernia, Nuno, Diaz, \& Villegas, 2014), (Li Vigni, y otros, 2015), y (Robinson Rodríguez, Jutinico Alarcón, \& Jiménez Moreno, 2013).

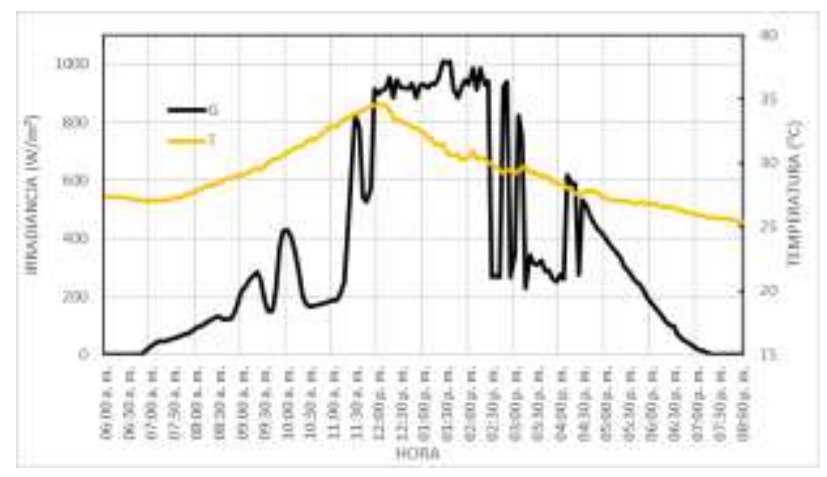

Gráfica 1 G y T medidos el día 14 de abril de 2019 Fuente: Elaboración Propia

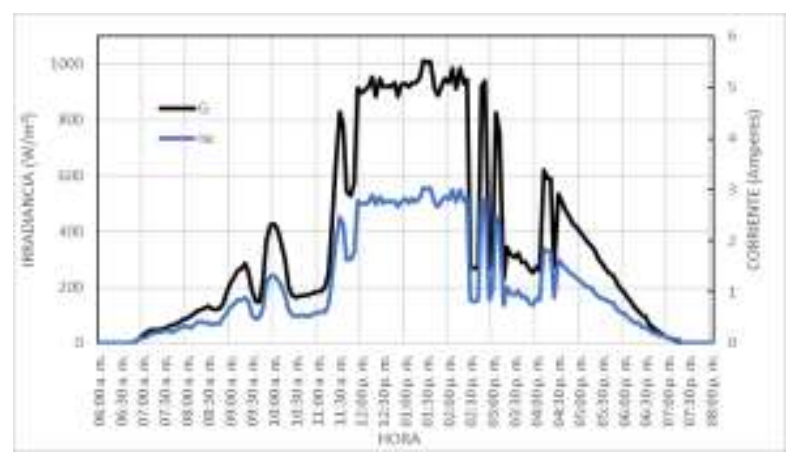

Gráfica 2 G e Isc medidos el día 14 de abril de 2019 Fuente: Elaboración Propia 


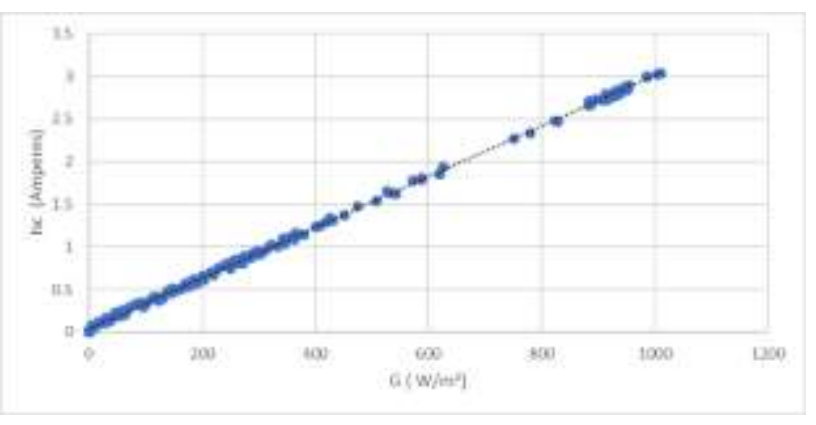

Gráfica 3 Curva de dispersión entre Isc y G. Datos medidos el día 14 de abril de 2019

Fuente: Elaboración Propia

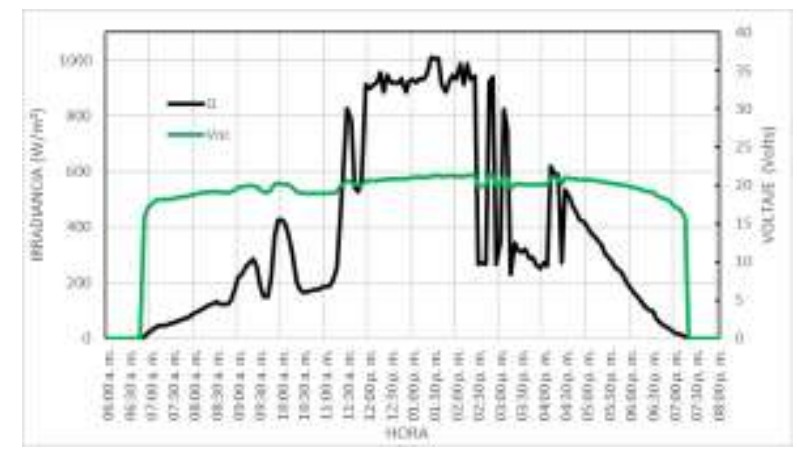

Gráfica 4 Voc y G medidos el día 14 de abril de 2019 Fuente: Elaboración Propia

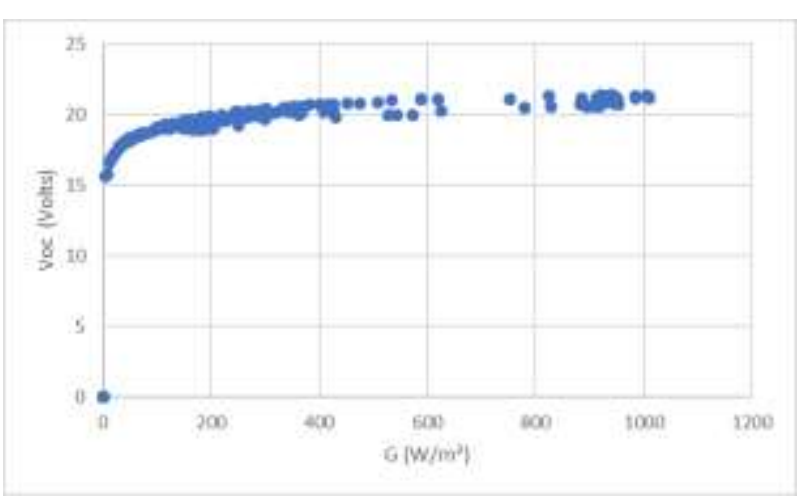

Gráfica 5 Curva de dispersión entre Voc y G. Datos medidos el día 14 de abril de 2019

Fuente: Elaboración Propia

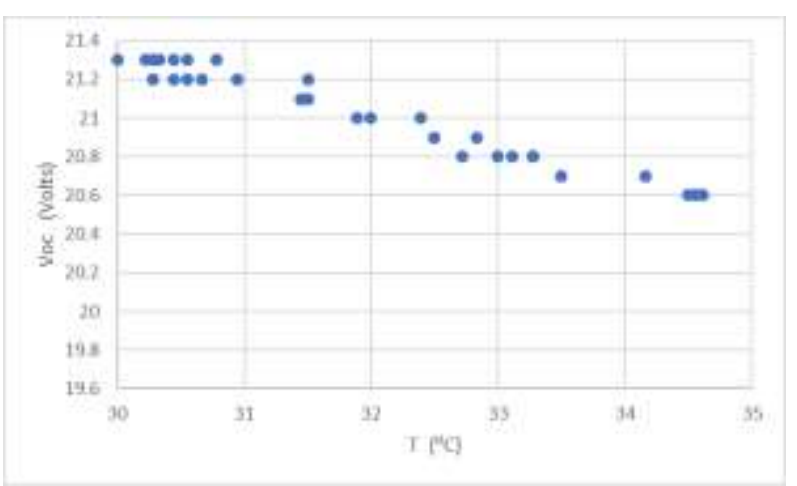

Gráfica 6 Curva de dispersión de Voc y T, de entre las 12:00 y 14:30 hrs., medidos el día 14 de abril de 2019 Fuente: Elaboración Propia

\section{Conclusiones}

Se ha diseñado un sistema de monitoreo de un arreglo fotovoltaico de paneles solares interconectados usando una red CAN, en el cual la información de tensión y corriente de salida, temperatura, son enviados a un controlador de señales digitales (DSC) para su posterior procesamiento. La red CAN ha sido construida con dispositivos de la familia Microchip. Se ha diseñado un programa para integración de los datos a un archivo .txt para el tratamiento de la información en Excel de Microsoft. Se ha validado la información obtenida mediante un análisis de comportamiento de la relación de las variables usando una base de conocimiento tomada de distintos autores en la literatura.

Con la información obtenida con este sistema es posible procesar los datos obtenidos para analizar diferentes escenarios incluyendo tópicos relacionados a la reconfiguración en la conexión de los paneles, estudios de eficiencia bajo distintos tipos de carga, pronóstico energético, y respuesta de la red a transitorios.

Dada la flexibilidad del sistema, también es posible crear redes mixtas e interconectar con otro tipo de redes, por ejemplo, inalámbricas que utilizan módulos XBee® bajo el estándar ZigBee®.

\section{Agradecimientos}

Agradecemos al Tecnológico Nacional de México / I. T. Mérida por todo el apoyo referente a los instrumentos y equipos de medida, y a la Facultad de Ingeniería de la Universidad Autónoma de Yucatán por el acceso a las instalaciones de su Laboratorio de Energías Renovables.

\section{Referencias}

Allegro. (2013). ACS714. Fully integrated Hall effect based linear current sensor datasheet. Worcester, Massachusetts. USA: Allegro microsystems.

Bosch, R. (1991). CAN specificationversion 2.0. Stuttgart, Germany.

Burr-Brown Corporation. (2020). INA114AP Precision instrumentation amplifier Datasheet. Dallas, Texas, USA.: Texas Instruments. 
Castillo Atoche, A., Vázquez Castillo, J., Ortegón Aguilar, J., Carrasco Álvarez, R., Sandoval Gío, J., \& Colli Menchi, A. (2015). A high-accuracy photovoltaic emulator system using ARM processors. Solar Energy, 389-398.

Kang, M., Kang, H., \& Choi, E. (2013). Monitoring technology available for measuring multiple-photovoltaic panel arrays. Proceedings of the TENCON Spring Conference, (págs. 1719). Sydney, Autralia.

Kaundal, V., Mondal, A., Sharma, P., \& Bansal, K. (2015). Tracing of shading effect on underachieving SPV cell of an SPV grid using wireless sensor network. Engineering Science and Technology, an International Journal, 475484.

Kekre, A., \& Gawre, S. K. (2017). Solar photovoltaic remote monitoring system using IOT. International Conference on Recent Innovations in Signal processing and Embedded Systems (RISE) (págs. 619-623). Bhopal, India: IEEE.

Li Vigni, V., La Manna, D., Riva Sanseverino, E., Di Dio, V., Romano, P., Di Buono, P., . . . Giaconia, C. (2015). Proof of Concept of an Irradiance Estimation System for Reconfigurable Photovoltaic Arrays. Energies, 6641-6657.

Löper, P., Pysch, D., Richter, M., Hermle, M., Janz, S., Zacharias, M., \& Glunz, S. (2012). Analysis of the Temperature Dependence of the Open-Circuit Voltage. SiliconPV 2012 Conference. (págs. 135-142). Leuven, Belgium.: Elsevier Ltd.

Maxim Integrated Products, Inc. (2019). DSB18B20. Programmable resolution 1-Wire digital thermometer datasheet. San José, california. USA.: Maxim.

Microchip Technology Inc. (2007). MCP2551. High-Speed CAN Transceiver Data Sheet. Chandler, AZ. USA.: Microchip Technology Inc.

Microchip Technology Incorporated. (2007). dsPIC30F3014/4013 Data Sheet. Chandler, AZ, USA.: Microchip Technology Incorporated.
Mohanraj, M., Thottungal, R., \& Jaikumar, K. (2013). A CAN bus based system for monitoring and fault diagnosis in wind turbine. International Conference on Emerging Trends in VLSI, Embedded System, Nano Electronics and Telecommunication System (ICEVENT) (págs. 1-3.). Tiruvannamalai, India: IEEE.

Núñez, J., Benítez, I., Proenza, R., Vázquez, L., \& Diaz, D. (2020). Metodología de diagnóstico de fallos para sistemas fotovoltaicos de conexión a red. Revista Iberoamericana de Automática e Informática Industrial, 94-105.

Papageorgas, P., Piromalis, D., Antonakoglou, K., Vokas, G., Tseles, D., \& Arvanitis, K. (2013). Smart Solar Panels: In-situ monitoring of photovoltaic panels based on wired and wireless sensor networks . Energy Procedia, 535-545.

Prieto, M., Pernia, A., Nuno, F., Diaz, J., \& Villegas, P. (2014). Development of a Wireless Sensor Network for Individual Monitoring of Panels in a Photovoltaic Plant. Sensors, 23792396.

Rivai, A., \& Rahim, N. (2013). A low-cost photovoltaic (PV) array monitoring system. Proceedings of the 2013 IEEE Conference on Clean Energy and Technology (CEAT) (págs. 169-174). Lankgkawi, Malaysia: IEEE.

Robinson Rodríguez, D., Jutinico Alarcón, A., \& Jiménez Moreno, R. (2013). Monitoring system for global solar radiation, temperature, current and power for a photovoltaic system interconnected with the electricity distribution network in Bogota. Proceedings of the 56th International Midwest Symposium on Circuits and Systems (págs. 4-7). Columbus, OH, USA: IEEE.

Sandoval Gío, J., Luján Ramírez, C., Sandoval Curmina, V., \& Chan Sosa, I. (2018). Sistema para el monitoreo remoto y análisis estadístico de la información energética disponible para optimizar el uso de arreglos de paneles solares. Revista de Tecnologías Computacionales, 1017.

Sarswat, S., Yadav, I., \& Maurya, S. (2019). Real Time Monitoring of Solar PV parameter using IoT. International Journal of Innovative Technology and Exploring Engineering (IJITEE), 267-271. 
Shariar, K., Mohammed, M., Rahman, M., \& Islam, M. (2011). Fabrication \& Characterization of Natural Dye Sensitized Solar Cell (NDSC). Gazipur, Bangladesh.: University of Technology,Gazipur,Bangladesh.

So, J., Jung, Y., Yu, G., \& Choi, J. (2007). Performance results and analysis of $3 \mathrm{~kW}$ gridconnected PV systems. Renew Energy, 18581872.

Solar World AG. (2012). Sunmodule SW 50 poly RMA data sheet. USA: Solar World AG.

Stoneking, R. (2002). A Simple CAN Node Using the MCP2510 and PIC12C67X. Chandler, AZ. USA.: Microchip Technology Inc.

Xiao, C., Yu , X., Yang, D., \& Que, D. (2014). Impact of solar irradiance intensity and temperature on the performance of compensated crystalline silicon solar cells. Solar Energy Materials and Solar Cells, 427-434.

Xie, Z., Yang, W., Zhang, X., \& Li, Z. (2015). The Study of Solar Monitoring System. International Forum on Energy, Environment Science and Materials (IFEESM 2015) (págs. 164-168). Shenzhen, China: Atlantis Press. 